\title{
Resonant light trapping in ultrathin films for water splitting
}

\author{
Hen Dotan ${ }^{1}$, Ofer Kfir ${ }^{2}$, Elad Sharlin ${ }^{1}$, Oshri Blank${ }^{1}$, Moran Gross ${ }^{1}$, Irina Dumchin ${ }^{1}$, Guy Ankonina ${ }^{3}$ \\ and Avner Rothschild ${ }^{1 \star}$
}

\begin{abstract}
Semiconductor photoelectrodes for solar hydrogen production by water photoelectrolysis must employ stable, non-toxic, abundant and inexpensive visible-light absorbers. Iron oxide $\left(\alpha-\mathrm{Fe}_{2} \mathrm{O}_{3}\right)$ is one of few materials meeting these requirements, but its poor transport properties present challenges for efficient charge-carrier generation, separation, collection and injection. Here we show that these challenges can be addressed by means of resonant light trapping in ultrathin films designed as optical cavities. Interference between forward- and backward-propagating waves enhances the light absorption in quarter-wave or, in some cases, deeper subwavelength films, amplifying the intensity close to the surface wherein photogenerated minority charge carriers (holes) can reach the surface and oxidize water before recombination takes place. Combining this effect with photon retrapping schemes, such as using V-shaped cells, provides efficient light harvesting in ultrathin films of high internal quantum efficiency, overcoming the trade-off between light absorption and charge collection. A water photo-oxidation current density of $4 \mathrm{~mA} \mathrm{~cm}^{-2}$ was achieved using a V-shaped cell comprising $\sim 26-\mathrm{nm}$-thick Ti-doped $\alpha-\mathrm{Fe}_{2} \mathrm{O}_{3}$ films on back-reflector substrates coated with silver-gold alloy.
\end{abstract}

T he efficient conversion of solar energy to hydrogen by means of water photoelectrolysis is a long-standing challenge with promise for solar energy conversion and storage in the form of synthetic fuels (so-called solar fuels) ${ }^{1,2}$. Important advances in studying water photoelectrolysis by semiconductor photoelectrodes $^{3}$ have been achieved in the past four decades, since the seminal report ${ }^{4}$ on photoinduced water splitting using $\mathrm{TiO}_{2}$ photoanodes. Despite these advances and intensive research and development efforts worldwide no photoelectrochemical system for solar hydrogen production has met the required performance benchmarks for efficiency, durability and cost. Numerous photoelectrodes were examined, but most of them were ruled out owing to poor stability or low efficiency ${ }^{5,6} \cdot \alpha-\mathrm{Fe}_{2} \mathrm{O}_{3}$ (haematite) is one of few materials with a favourable combination of stability in aqueous solutions, visible-light absorption, nontoxicity, abundance and low $\operatorname{cost}^{7-9}$. With an energy bandgap of $2.1 \mathrm{eV}, \alpha-\mathrm{Fe}_{2} \mathrm{O}_{3}$ photoanodes can theoretically reach water photooxidation current densities as high as $12.6 \mathrm{~mA} \mathrm{~cm}^{-2}$ under air mass 1.5 global (AM1.5G) solar irradiation conditions ${ }^{10}$, potentially enabling a maximum solar to hydrogen conversion efficiency of $15.5 \%$ to be reached in an ideal tandem cell configuration ${ }^{11}$. However, because of the low internal quantum efficiency (IQE), only a quarter of the theoretical limit has been achieved by the best $\alpha-\mathrm{Fe}_{2} \mathrm{O}_{3}$ photoanode reported so far ${ }^{12}$.

The low IQE of $\alpha-\mathrm{Fe}_{2} \mathrm{O}_{3}$ photoanodes has been attributed to the slow water oxidation kinetics ${ }^{13}$ and short diffusion length of the photogenerated minority charge carriers (holes) ${ }^{14}$ resulting in significant loss due to electron-hole recombination. Extensive efforts have been directed towards enhancing the water oxidation kinetics using catalysts ${ }^{7,9,12}$, and reducing bulk recombination by developing nanostructured architectures that decouple the charge transport and optical path lengths ${ }^{7-9,15}$. Nanostructuring thick layers has been the main route to balance the trade-off between light absorption and collection of photogenerated holes ${ }^{8,9,15}$. This tradeoff limits the solar energy conversion efficiency of semiconductors with poor transport properties wherein the minority chargecarrier collection length is smaller than the light penetration depth $^{16}$. Despite these efforts, state-of-the-art nanostructured $\alpha-\mathrm{Fe}_{2} \mathrm{O}_{3}$ photoanodes still exhibit excessive bulk recombination that consumes three quarters, or more, of the photogenerated charge carriers. On the other hand, the injection yield of holes that have reached the surface exceeds $90 \%$ under sufficiently high anodic potentials ${ }^{17}$. Recent studies on thick layers ${ }^{18,19}$ as well as ultrathin film ${ }^{20}$ confirm that the efficiency of $\alpha-\mathrm{Fe}_{2} \mathrm{O}_{3}$ photoanodes is mainly limited by the collection of photogenerated holes at the surface. Thus, reducing bulk recombination is the key to improving the IQE of $\alpha-\mathrm{Fe}_{2} \mathrm{O}_{3}$ photoanodes-an important step towards efficient, sustainable, durable and potentially inexpensive solar hydrogen production.

Whereas the conventional approach for balancing photogeneration and collection of minority charge carriers at the surface of $\alpha-\mathrm{Fe}_{2} \mathrm{O}_{3}$ photoanodes centres around nanostructured porous thick layers $(\geq 0.5 \mu \mathrm{m})$ of high surface area, here we show a radically different approach in which compact flat ultrathin films are designed as light trapping structures. Such films can be easily produced using thin-film deposition methods, and they do not have a high surface area that enhances surface recombination and may lower the surface photovoltage- - the driving force for photoelectrochemical processes on semiconductor photoelectrodes ${ }^{21}$. Instead of decoupling the optical and charge transport path lengths we exploit the wave-nature of light propagation in subwavelength structures to tailor the light intensity distribution inside the film, amplifying the intensity close to the electrode/electrolyte interface. This enables maximization of the absorption in regions where the

\footnotetext{
${ }^{1}$ Department of Materials Science and Engineering, Technion-Israel Institute of Technology, Haifa 32000, Israel, ${ }^{2}$ Physics Department, Technion-Israel Institute of Technology, Haifa 32000, Israel, ${ }^{3}$ Photovoltaics Laboratory, Technion-Israel Institute of Technology, Haifa 32000, Israel.

*e-mail: avnerrot@technion.ac.il.
} 
photogenerated minority charge carriers can reach the surface and minimization of the wasted absorption in regions where they are lost to recombination.

\section{Ultrathin film $\alpha-\mathrm{Fe}_{2} \mathrm{O}_{3}$ photoanodes}

The rationale for using ultrathin $(<50 \mathrm{~nm})$ film $\alpha-\mathrm{Fe}_{2} \mathrm{O}_{3}$ photoanodes stems from their high IQE compared with their thick-layer counterparts $^{22}$. This is demonstrated in Supplementary Fig. S1, which shows that the charge separation and collection yield of photogenerated holes at the surface of Ti-doped $\alpha-\mathrm{Fe}_{2} \mathrm{O}_{3}$ thin films (Supplementary Fig. S2) increases with decreasing film thickness, reaching $58 \pm 6 \%$ for the thinnest film $(16 \pm 5 \mathrm{~nm})$. This exceeds the collection yield found in state-of-the-art nanostructured Si-doped $\alpha-\mathrm{Fe}_{2} \mathrm{O}_{3}$ thick layers ${ }^{17}$ by a factor of three, approximately, demonstrating the potential advantage of ultrathin films of high crystalline quality (see Supplementary Figs S4-S8). The salient problem is that their optical density is too small. The thinnest film, that is the one with the highest collection yield, is nearly translucent (see Supplementary Fig. S2-C). Thus, effective utilization of ultrathin $\alpha-\mathrm{Fe}_{2} \mathrm{O}_{3}$ films requires special means to enhance the probability of lightmatter interaction in subwavelength structures. The standard approach for trapping light in thin film solar cells works only for films much thicker than a half wavelength ${ }^{23}$. This is too thick for $\alpha-\mathrm{Fe}_{2} \mathrm{O}_{3}$ because of the small collection length of the photogenerated holes in this material ${ }^{9,13,14}$. To address this challenge we employ a resonant light trapping strategy that surpasses the classical limits of statistical ray optics ${ }^{24}$, enabling efficient photon harvesting well below the minimum film thickness required for the standard approach.

\section{Resonant light trapping in ultrathin films}

Our strategy employs optical cavities comprising ultrathin absorbing films on reflective substrates serving as current collectors and back reflectors that give rise to interference between the forwardand backward-propagating waves, as illustrated schematically in Fig. 1. This enhances the absorption by increasing the photon lifetime in the film, reaching maximum absorption in the cavity resonance modes. For normal incidence on an ideal cavity the first resonance mode occurs in quarter-wave films, that is, when the film thickness $(d)$ is a quarter of the wavelength $(\lambda)$ of the light inside the film. We note that $\lambda=\lambda_{0} n_{2}^{-1}$, where $\lambda_{0}$ is the wavelength in vacuum and $n_{2}$ is the refractive index of the film. The quarter-wave condition takes into account the $\pi$ phase shifts on reflection from the surface of the film $(x=0)$ and from the film/substrate interface $(x=d)$, and another $\pi$ phase shift on traversing the film forth and back. Consequently, the first-order reflection is in anti-phase with the higher-order reflections, as shown in Fig. 1, thereby suppressing the intensity of the back-reflected light. Moreover, the light intensity increases near the surface owing to constructive interference between the forward- and backward-propagating waves. For other conditions such as oblique light or optical cavities employing metallized substrates with complex refractive indices the optimal film thickness is smaller than a quarter-wave, providing another degree of freedom for optimization.

The quarter-wave resonance condition is well defined for monochromatic light, but our goal is to harvest as many photons as possible from the incident sunlight in a broad spectral range between the absorption edge of the absorber $\left(\lambda_{0}^{\max }=590 \mathrm{~nm}\right.$ for $\left.\alpha-\mathrm{Fe}_{2} \mathrm{O}_{3}\right)$ and the fall-off of the sunlight spectrum $\left(\lambda_{0}^{\mathrm{min}}=300 \mathrm{~nm}\right)$. Therefore, we should find the optimal film thickness for trapping sunlight in this spectral range. Moreover, owing to the strong dependence of the collection efficiency of photogenerated minority charge carriers on the distance from the surface $e^{25}$ we must take into account not only how many photons are absorbed but also where they are absorbed. The photocurrent density can then be calculated by integrating the product of the photogeneration distribution, which scales with the squared optical electric field strength ${ }^{26}$, and

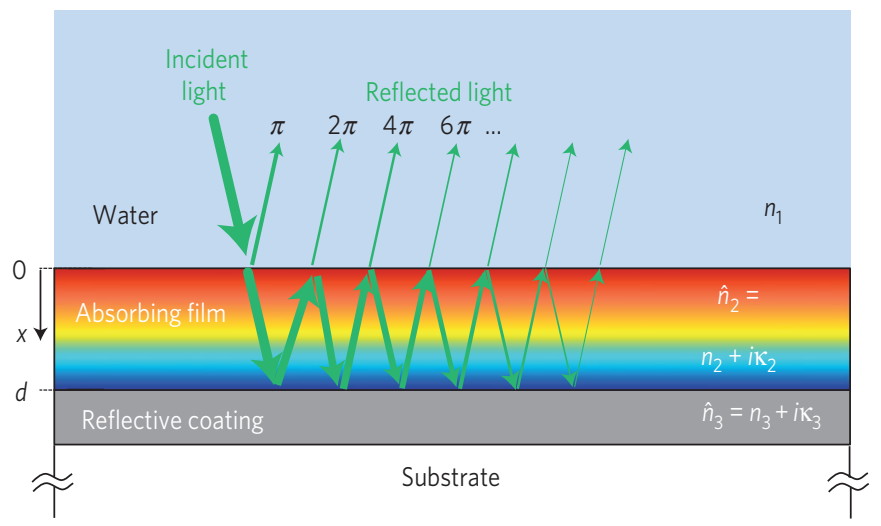

Figure 1 | Resonant light trapping in quarter-wave films. Schematic illustration of the light propagation in a quarter-wave $(d=\lambda / 4$, for normal incidence) absorbing film on a back-reflector substrate. The different colours represent the light intensity distribution across the film (red, high; blue, low).

the IQE distribution across the film over the entire film thickness and over the solar irradiance spectrum.

To calculate the light intensity distribution inside the film we take the plane-wave solution of Maxwell's electromagnetic wave equation and tailor it to fit the boundary conditions of our system with incident solar radiation, at AM1.5G conditions, striking the optical stack illustrated in Fig. 1. From these solutions we obtain analytical expressions (see Supplementary Equations S1-S3) for the spectral photon flux profiles (the number of photons of wavelength $\lambda_{0}$ reaching a distance $x$ from the surface per unit time, unit area and unit wavelength) $I_{\lambda_{0}}\left(\lambda_{0}, x\right)$ for normal incidence on $\alpha-\mathrm{Fe}_{2} \mathrm{O}_{3}$ films on ideally reflective, transparent or partially reflective metallized substrates with complex refractive indices $\hat{n}_{3}=n_{3}+i \kappa_{3}$, where $n_{3}$ and $\kappa_{3}$ are the refractive and attenuation indices, respectively. The metallized substrates give rise to complex reflection coefficients at the film/substrate interface ${ }^{27}, \hat{r}_{23}=\left(\hat{n}_{2}-\hat{n}_{3}\right)\left(\hat{n}_{2}+\hat{n}_{3}\right)^{-1}$. By integrating $I_{\lambda_{0}}\left(\lambda_{0}, x\right)$ we obtain the photon flux profiles, $I(x)=\int_{\lambda_{0}^{\min }}^{\lambda_{\max }^{\max }} I_{\lambda_{0}}\left(\lambda_{0}, x\right) \mathrm{d} \lambda_{0}$, as a function of depth $(x)$ inside the film. Photon flux profiles predicted for Ti-doped $\alpha-\mathrm{Fe}_{2} \mathrm{O}_{3}$ films on ideally reflective $\left(\hat{r}_{23}=-1\right)$, transparent $\left(\hat{r}_{23}=0\right)$ and metallized substrates coated with silver or platinum are shown in Fig. 2. Further profiles for films on aluminium- or gold-coated substrates are presented in Supplementary Fig. S26. The optical constants of the Ti-doped $\alpha-\mathrm{Fe}_{2} \mathrm{O}_{3}$ films were measured by spectroscopic ellipsometry (see Supplementary Fig. S11) and the constants for the metallized substrates were taken from the ellipsometer database.

The predicted photon flux profiles for films on reflective substrates exhibit a periodic dependence on the film thickness. The first resonance mode of the ideal cavity is predicted to occur at a film thickness of $43 \mathrm{~nm}$, reaching the maximal intensity at the surface. Partially reflective metallized substrates give rise to smaller photon fluxes, due to absorption in the metal coating, and the resonance modes are shifted to smaller film thicknesses due to phase shifts larger than $\pi$ at the film/substrate interface (see Supplementary Fig. S25). The optical loss due to absorption in the metal coating is low for silver and aluminium, but quite high for platinum and gold. The first resonance mode is predicted to occur at a film thickness of 20,20,24 or $30 \mathrm{~nm}$ for silver, gold, platinum or aluminium coatings, respectively. Thus, the selection of the reflective coating material influences the optimal film thickness. All in all, considerably larger photon fluxes are predicted in films, of the optimal thickness, on reflective substrates compared with their counterparts on transparent substrates wherein the photons have only one pass through the film and their flux 
a

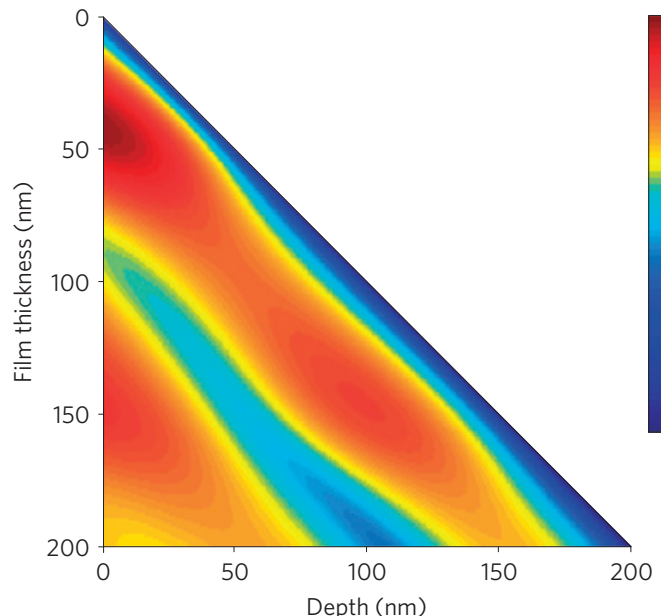

C

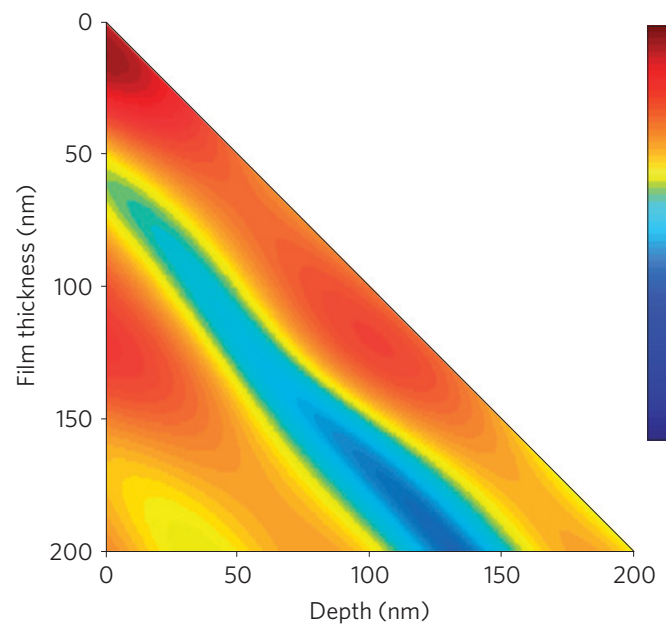

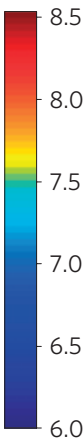
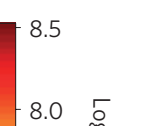

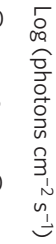

b
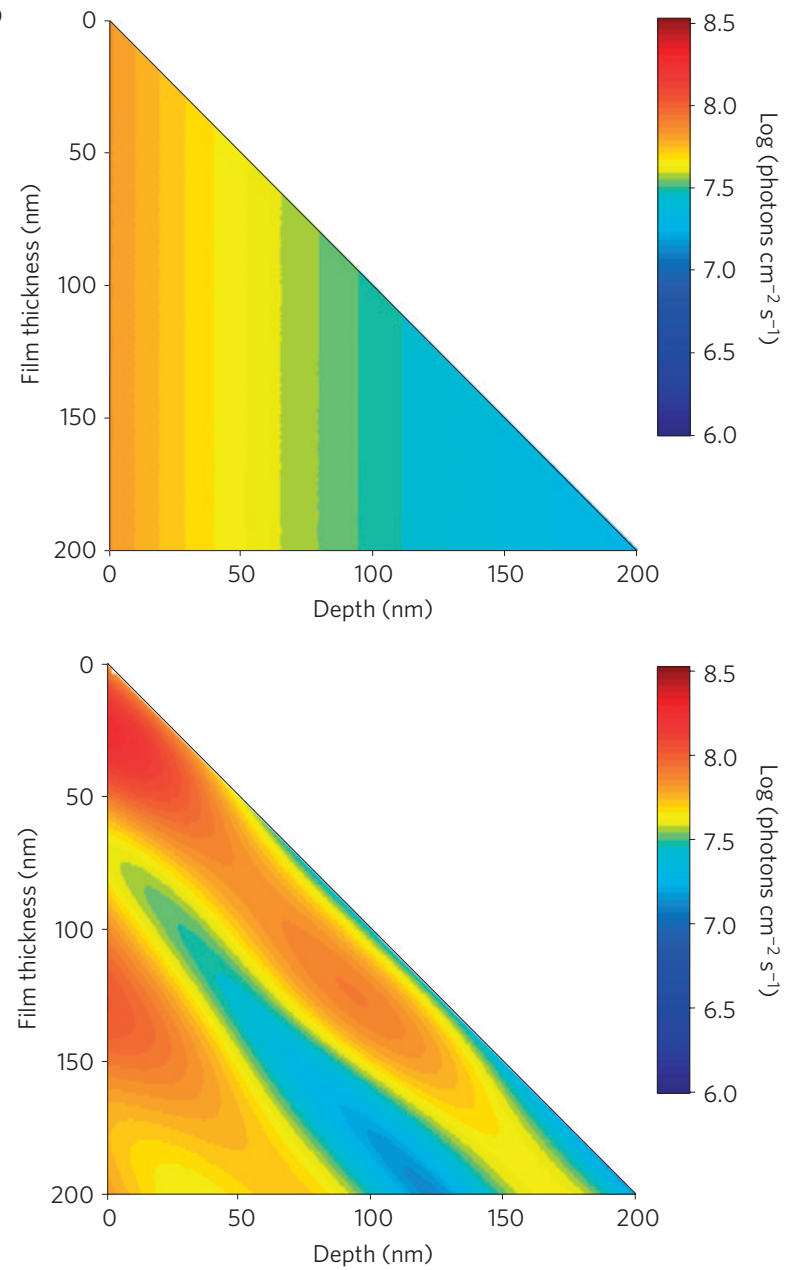

Figure 2 | Light intensity maps. a-d, Predicted photon flux profiles as a function of film thickness and depth from the surface into the film for Ti-doped $\alpha-\mathrm{Fe}_{2} \mathrm{O}_{3}$ films on ideally reflective (a), transparent (b) and metallized substrates coated with silver (c) or platinum (d).

decreases exponentially with $x$ according to Lambert's law with no dependence on the film thickness.

Figure 2 shows that in the first resonance mode high photon fluxes are concentrated close to the surface. This is critical for efficient collection of the photogenerated minority charge carriers, enabling them to reach the surface and drive the water splitting reaction before bulk recombination takes place. Furthermore, the photon flux close to the film/substrate interface is suppressed in films on ideally reflective substrates because of the $\pi$ phase shift on reflection from an ideal reflector ${ }^{27}$. This is expected to reduce the loss due to backward injection of minority charge carriers to the substrate, which has been identified as a major source of loss in ultrathin $\alpha-\mathrm{Fe}_{2} \mathrm{O}_{3}$ photoanodes ${ }^{28}$.

To verify our model calculations we deposited Ti-doped $\alpha-\mathrm{Fe}_{2} \mathrm{O}_{3}$ films of different thicknesses between 8 and $155 \mathrm{~nm}$ on platinized and, for comparison, fluorinated tin oxide (FTO)-coated glass substrates (see Supplementary Fig. S2). The absorptance spectra, $a\left(\lambda_{0}\right)$, of the specimens were obtained from reflection and transmission measurements (Supplementary Fig. S12). The absorbed photon flux was calculated using the formula ${ }^{29} I_{\text {abs }}=\int_{\lambda_{0}^{\min }}^{\lambda_{\max }} I_{\lambda_{0}}^{\text {Sun }}\left(\lambda_{0}\right) a\left(\lambda_{0}\right) \mathrm{d} \lambda_{0}$, where $I_{\lambda_{0}}^{\text {Sun }}$ is the solar irradiance spectrum at AM1.5G conditions. The results are shown in Fig. 3 in terms of $I_{\text {abs }}$ and the photogenerated current density, $J_{\mathrm{pg}}=q I_{\mathrm{abs}}$, where $q$ is the elementary charge. The black squares show the absorption in the platinized specimens. The model calculations, shown by the dashed line black curve, nicely fit the experimental results. We note that the absorptance obtained by these measurements includes contributions from both the $\alpha-\mathrm{Fe}_{2} \mathrm{O}_{3}$ film and the platinized substrate, and we cannot extract directly the net absorption in the $\alpha-\mathrm{Fe}_{2} \mathrm{O}_{3}$ film. Instead, we show (full line black curve) the predicted absorption in the $\alpha-\mathrm{Fe}_{2} \mathrm{O}_{3}$ films, obtained by integrating the product of the photon flux profiles in Fig. $2 \mathrm{~d}$ and the absorption coefficient of the films (Supplementary Fig. S11) over the entire film thickness. Similar calculations for films on ideally reflective ( $R=1$, red curve), transparent $(R=0$, blue curve) and metallized substrates coated with silver (green curve), aluminium (grey curve) or gold (orange curve) are also shown in Fig. 3. For the translucent FTO-coated specimens we obtain the net absorptance in the Ti-doped $\alpha-\mathrm{Fe}_{2} \mathrm{O}_{3}$ films from transmission and reflection measurements of coated and uncoated specimens (see Supplementary Fig. S14). These results are shown by the blue circles in Fig. 3.

The absorption in $\alpha-\mathrm{Fe}_{2} \mathrm{O}_{3}$ films on platinized substrates is predicted to reach the first maximum at a film thickness of $36 \mathrm{~nm}$ with a photogenerated current density of $5.1 \mathrm{~mA} \mathrm{~cm}^{-2}$, that is $40 \%$ of the ultimate limit for $\alpha-\mathrm{Fe}_{2} \mathrm{O}_{3}$ (ref. 10). For comparison, the same film would absorb approximately half the photons $\left(2.8 \mathrm{~mA} \mathrm{~cm}^{-2}\right)$ if it were on a transparent substrate. Calculations for films on ideally reflective substrates (red curve) show that a 47-nm-thick film would reach a photogenerated current density of $8.9 \mathrm{~mA} \mathrm{~cm}^{-2}$, that is $71 \%$ of the theoretical limit. This demonstrates the effectiveness of our light trapping strategy, as the same film on a transparent substrate yields only $3.4 \mathrm{~mA} \mathrm{~cm}^{-2}$. Thus, the photon harvesting yield can be nearly tripled by replacing the ubiquitous transparent substrates with suitable back reflectors. Similar calculations for films on silver, 


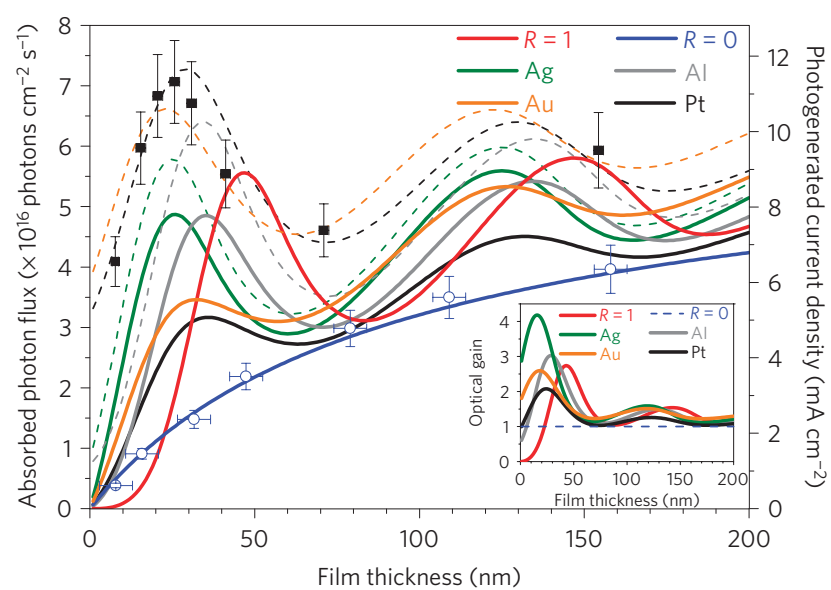

Figure 3 | Sunlight absorption in $\alpha-\mathrm{Fe}_{2} \mathrm{O}_{3}$ films on back-reflector substrates versus their counterparts on transparent substrates. Absorbed photon flux and the corresponding photogenerated current density as a function of film thickness for specimens comprising Ti-doped $\alpha-\mathrm{Fe}_{2} \mathrm{O}_{3}$ films on platinized (black squares) or FTO-coated substrates (blue circles). The error bars represent the range of (estimated) systematic errors in the measurements. The dashed curves show the predicted absorption in the entire specimen, comprising contributions from both film and substrate; the solid line curves show the predicted net absorption in the $\alpha-\mathrm{Fe}_{2} \mathrm{O}_{3}$ films. The inset shows the predicted optical gain, that is, the amount of photons absorbed in $\alpha-\mathrm{Fe}_{2} \mathrm{O}_{3}$ films on different substrates divided by the absorption in films of the same thickness on FTO-coated substrates.

aluminium- and gold-coated substrates are predicted to yield high optical gains (see Fig. 3, inset), with a maximum gain of 4.2 for a 16-nm-thick film on a silver-coated substrate (green curve). It is noteworthy that the rest of the photons that are not absorbed in the $\alpha-\mathrm{Fe}_{2} \mathrm{O}_{3}$ film are not necessarily lost. The back-reflected photons that escape can be collected using photon retrapping schemes such as using $\mathrm{V}$-shaped cells ${ }^{30}$, thereby further boosting the absorption.

\section{From light trapping to water photo-oxidation}

The photocurrent density, $J_{\text {photo }}$, is a product of the photogeneration rate per unit time and unit volume at distance $x$ from the surface, $g(x)$, and the IQE distribution function, $P(x)$, integrated over the entire film thickness: $J_{\text {photo }}(d)=q \int_{0}^{d} g(x) P(x) \mathrm{d} x$. The photogeneration rate is a product of the spectral photon flux distribution inside the film, $I_{\lambda_{0}}\left(\lambda_{0}, x\right)$, and the absorption coefficient of the absorber, $\alpha\left(\lambda_{0}\right)$, integrated over the wavelength range between the absorption edge and the fall-off of the sunlight spectrum $^{16}: g(x)=\int_{\lambda_{0}^{\min }}^{\lambda_{0}^{\max }} I_{\lambda_{0}}\left(\lambda_{0}, x\right) \alpha\left(\lambda_{0}\right) \mathrm{d} \lambda_{0} . P(x)$ is the probability for the photogenerated minority charge carriers to separate from the majority carriers, reach the surface and drive the water splitting reaction. Only those carriers that reach the front surface of the film and are forward injected to the electrolyte contribute to the water splitting process, whereas those reaching the backside of the film and being backward injected to the substrate reduce the photocurrent. Backward injection to the substrate may occur by interfacial recombination with charge carriers of the opposite charge arriving from the substrate (that is, the back electrode). We designate by $\Phi$ the probability for charge separation and transport in the forward direction, that is, minority charge carriers going towards the surface. The collection probability of minority charge carriers generated at a distance $x$ from the surface scales exponentially with $-x / L$, where $L$ is their collection length ${ }^{25}$. Designating by $\vec{P}_{\mathrm{F}}$ the probability for forward injection to the electrolyte, that is, the probability for minority charge carriers that have reached the surface to drive the desired electrochemical reaction, the fraction of photogenerated minority charge carriers

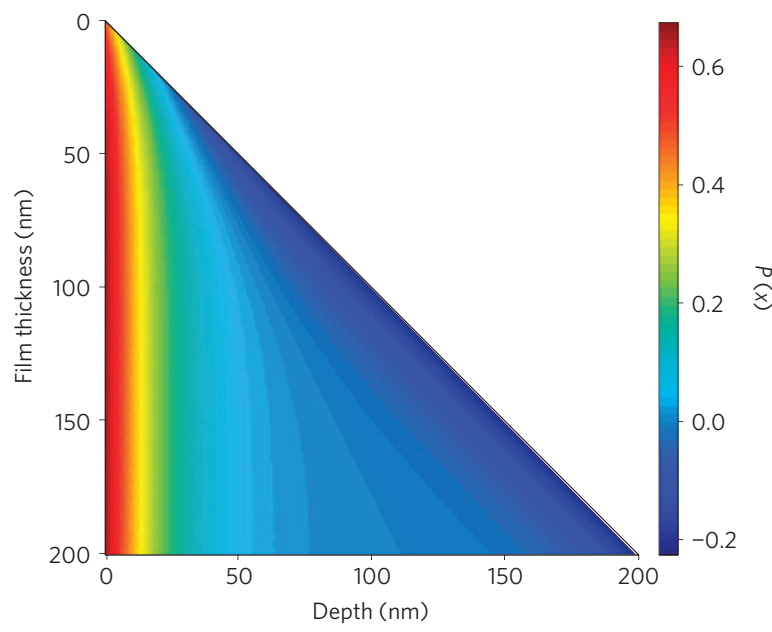

Figure 4 | Internal quantum efficiency map. The predicted IQE distribution inside the film as a function of film thickness and depth for $\alpha-\mathrm{Fe}_{2} \mathrm{O}_{3}$ films with $\Phi=0.75, \vec{P}_{\mathrm{F}}=\overleftarrow{P}_{\mathrm{B}}=0.9$ and $L=20 \mathrm{~nm}$

that end up with a positive contribution to the photocurrent is $\vec{P}_{\mathrm{F}}$ $\Phi \mathrm{e}^{-x / L}$. Likewise, the fraction of their counterparts ending up with a negative contribution due to backward injection to the substrate is $\overleftarrow{P}_{\mathrm{B}}(1-\Phi) \mathrm{e}^{-(d-x) / L}$, where $\overleftarrow{P}_{\mathrm{B}}$ is the probability for backward injection. All in all, $P(x)=\vec{P}_{\mathrm{F}} \Phi \mathrm{e}^{-x / L}-\overleftarrow{P}_{\mathrm{B}}(1-\Phi) \mathrm{e}^{-(d-x) / L}$. We note that this expression can be derived from the constituent equations of solar cells ${ }^{31}$.

$\vec{P}_{\mathrm{F}}, \overleftarrow{P}_{\mathrm{B}}, L$ and $\Phi$ depend on the doping level, preparation and operation conditions of the photoelectrode. Figure 4 shows $P(x)$ as a function of $d$ and $x$ for $\Phi=0.75, \vec{P}_{\mathrm{F}}=\overleftarrow{P}_{\mathrm{B}}=0.9$ and $L=20 \mathrm{~nm}$. These values were found to fit quite well the photocurrent densities obtained with our Ti-doped $\alpha-\mathrm{Fe}_{2} \mathrm{O}_{3}$ films on platinized substrates, and they are within range of the expected values ${ }^{13,17,32} \cdot P(x)$ reaches more than $60 \%$ close to the surface but it decays exponentially to near zero values deeper than $\sim 20 \mathrm{~nm}$ from the surface, reaching negative values close to the interface with the substrate.

Taking the product of $g(x)$, obtained using the calculated photon flux profiles in Fig. 2 and the measured absorption coefficient of our films (Supplementary Fig. S11), and $P(x)$ from Fig. 4 we obtain the predicted photocurrent density per unit volume profiles, $\mathrm{d} J_{\text {photo }} / \mathrm{d} x=q g(x) P(x)$, for films on ideally reflective, transparent or metallized substrates, as shown in Fig. 5 . These profiles show that mainly the front region down to $\sim 20 \mathrm{~nm}$ from the surface contributes to the photocurrent. The rest of the film is inactive, for the most part, owing to bulk recombination, whereas the backside of the film has a negative contribution due to backward injection to the substrate. The advantage of our optical cavity design is clearly demonstrated by the hotspots of high photocurrent density close to the surface of films whose thickness is close to the optimal thickness for the first resonance mode. The highest photocurrent densities are predicted for the ideal cavity, which is also effective in reducing the backward injection to the substrate by suppressing the light intensity close to the film/substrate interface (see Fig. 2a). Films on partially reflective metallized substrates also exhibit hotspots of high photocurrent densities, but the hotspots become weaker in going from highly reflective metals such as silver to poorly reflective ones such as platinum. We also note that the calculations suggest that the metallized substrates do not reduce the backward-injection loss as effectively as their ideally reflective counterparts.

The photocurrent density per unit area, $J_{\text {photo }}$, is calculated by integrating the photocurrent density per unit volume over the entire film thickness. Figure 6a shows the predicted photocurrent density as a function of film thickness for $\alpha-\mathrm{Fe}_{2} \mathrm{O}_{3}$ films on different substrates, assuming ideal forward-injection conditions 


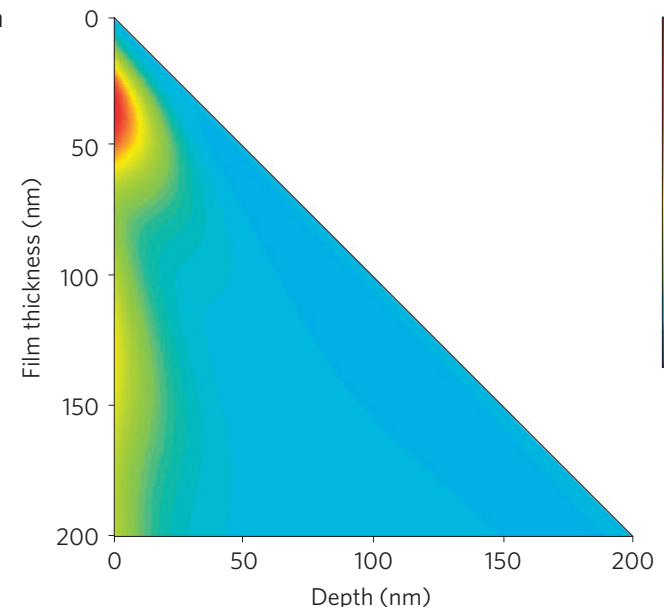

C

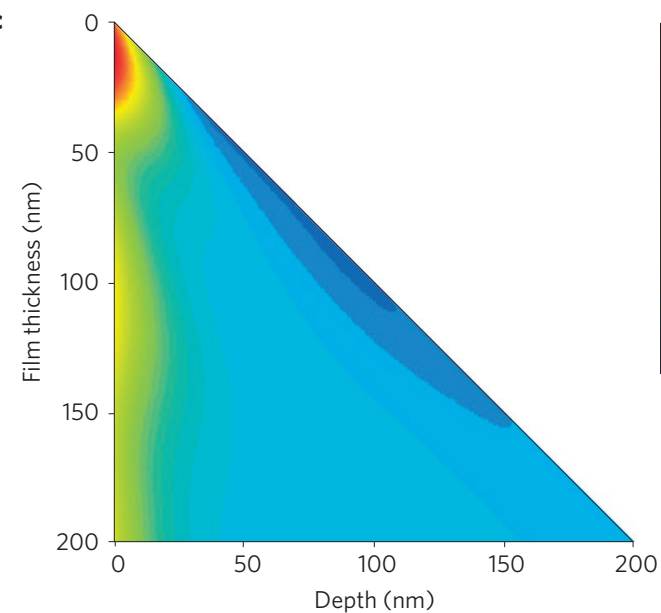

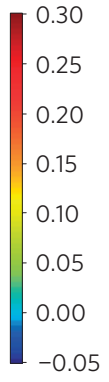

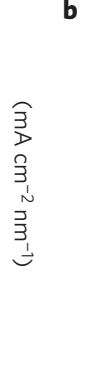

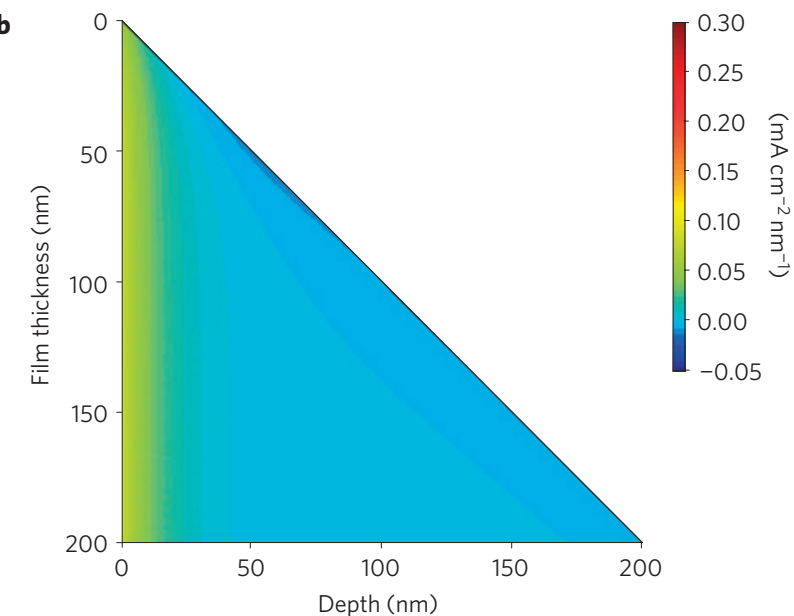

d

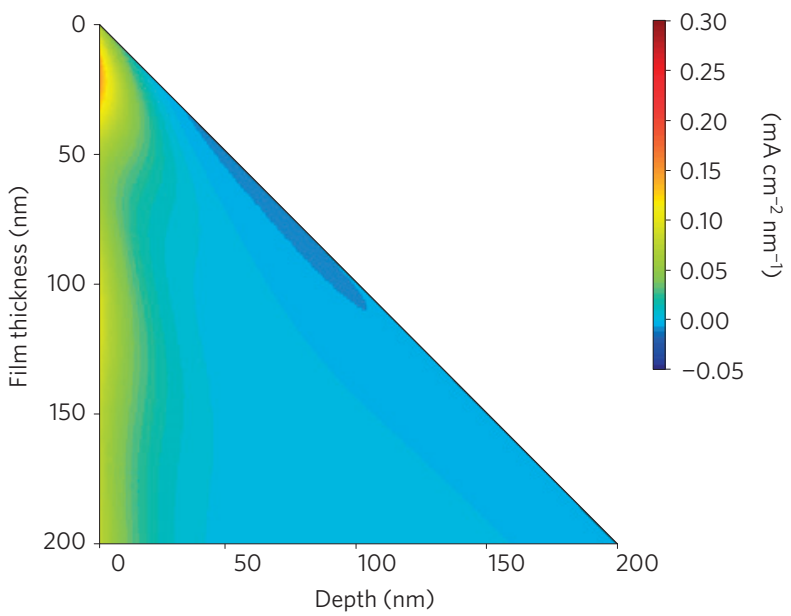

Figure 5 | Photocurrent distribution maps. a-d, Predicted photocurrent density distribution maps as a function of film thickness and depth for $\alpha$-Fe ${ }_{2} \mathrm{O}_{3}$ films on ideally reflective (a), transparent (b) and metallized substrates coated with silver (c) or platinum (d). Further profiles for aluminium- and gold-coated substrates are presented in Supplementary Fig. S27.

(that is, $\Phi=1$ and $\vec{P}_{\mathrm{F}}=1$ ). Such conditions may be realized using sufficiently high potentials ${ }^{17}$ and selective transport layers to block the backward injection to the substrate ${ }^{33}$. Films on reflective substrates are predicted to yield maximum photocurrents close to the first resonance modes of the respective optical cavities. The maxima are quite narrow and therefore the film thickness must be precisely tuned to achieve the optimal performance, as an offset of just a few nanometres significantly decreases the photocurrent. A maximum of $4.8 \mathrm{~mA} \mathrm{~cm}^{-2}$ is predicted for a 43 -nm-thick film on an ideally reflective substrate (red curve). This value exceeds the record obtained with the best $\alpha-\mathrm{Fe}_{2} \mathrm{O}_{3}$ photoanode reported so far $^{12}$, demonstrating the potential advantage of our approach. Photocurrent densities of $4.6,4.3,3.1$ and $2.9 \mathrm{~mA} \mathrm{~cm}^{-2}$ are predicted for 22-, 31-, 24- and 29-nm-thick $\alpha-\mathrm{Fe}_{2} \mathrm{O}_{3}$ films on silver-, aluminium-, gold- and platinum-coated substrates, respectively. The predicted photocurrent gain with respect to films of the same thickness on transparent substrates is shown in the inset of Fig. 6a. Optical cavities comprising ultrathin $\alpha-\mathrm{Fe}_{2} \mathrm{O}_{3}$ films are predicted to exhibit considerable gains reaching 3.6, 2.8, 2.3 and 2.0 for 14-, 28-, 18 - and $24-n m-t h i c k$ films on silver-, aluminium-, gold- or platinum-coated substrates, respectively, whereas the gain for films on ideally reflective substrates reaches 2.9 for a 42-nm-thick film.

To verify our model calculations the photocurrent density of Ti-doped $\alpha-\mathrm{Fe}_{2} \mathrm{O}_{3}$ films on platinized or, for comparison, FTO-coated substrates was measured in a $1 \mathrm{M} \mathrm{NaOH}$ solution under $100 \mathrm{~mW} \mathrm{~cm}^{-2}$ white-light illumination. Figure $6 \mathrm{~b}$ shows the photocurrent density, measured at an applied potential of $1.4 \mathrm{~V}$ against the reversible hydrogen electrode $\left(V_{\mathrm{RHE}}\right)$, plotted as a function of the film thickness. Further results are shown in Supplementary Figs S15-S17. The photocurrent density reached a maximum of $1.39 \pm 0.03 \mathrm{~mA} \mathrm{~cm}^{-2}$ for a $26 \pm 2$-nm-thick Ti-doped $\alpha-\mathrm{Fe}_{2} \mathrm{O}_{3}$ film on a platinized substrate, 2.6 times higher than the photocurrent density obtained with a film of about the same thickness $(32 \pm 8 \mathrm{~nm})$ on an FTO-coated substrate. Moreover, this ultrathin film outperformed any of its counterparts on FTO-coated transparent substrates, including much thicker films. These results clearly demonstrate the advantage of our light trapping scheme, even though these proof-of-concept tests were carried out with structures far from the optimal design because of the high optical loss due to absorption in the platinum coating as well as other losses as described in the following.

The experimental results in Fig. $6 \mathrm{~b}$ were fitted with model calculations assuming $L=20 \pm 2 \mathrm{~nm}$, consistent with other reports $^{13,32}$, and taking $\vec{P}_{\mathrm{F}} \times \Phi$ and $\overleftarrow{P}_{\mathrm{B}} \times(1-\Phi)$ as fitting parameters. All the other parameters were obtained from optical measurements of the specimens (Supplementary Fig. S11). An excellent agreement was obtained with $\vec{P}_{\mathrm{F}} \times \Phi=0.70 \pm 0.05$ and $\overleftarrow{P}_{\mathrm{B}} \times(1-\Phi)=0.25 \pm 0.05$, validating our model calculations. The fitting results are consistent with $\vec{P}_{\mathrm{F}}=0.95 \pm 0.05$, close to the injection efficiency of our films as measured by the method described in ref. $17, \overleftarrow{P}_{\mathrm{B}}=1 \pm_{0.3}^{0}$, and $\Phi=0.74 \pm 0.07$. Thus, a significant fraction of the potentially achievable photocurrent predicted for these structures is lost for backward injection to the 
a

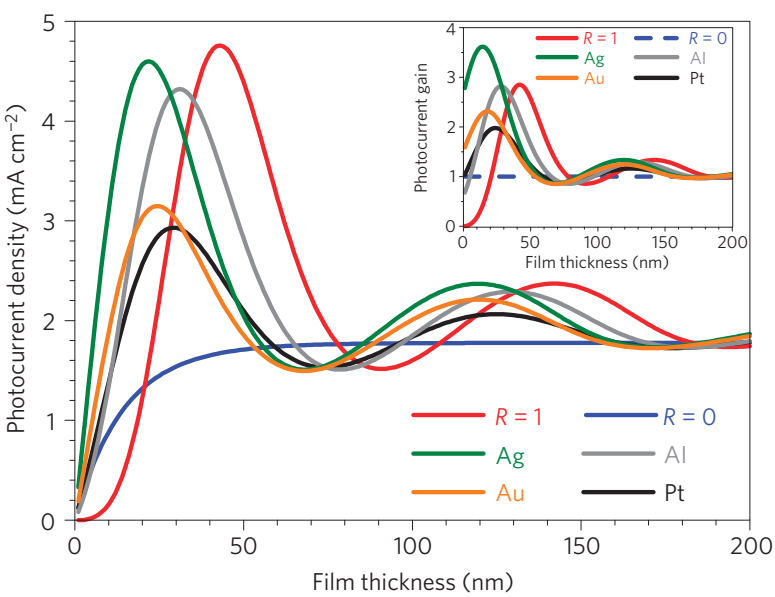

b

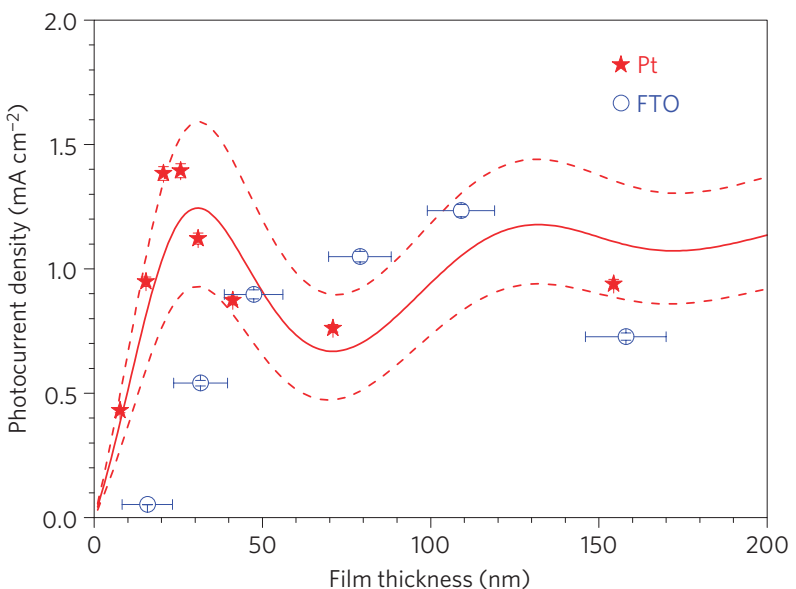

Figure 6 | Photocurrent as a function of film thickness. a, Predicted photocurrent density as a function of film thickness for $\alpha-\mathrm{Fe}_{2} \mathrm{O}_{3}$ films on different substrates under ideal forward-injection conditions.

b, Photocurrent density, measured at $1.4 V_{\mathrm{RHE}}$ in $100 \mathrm{~mW} \mathrm{~cm}^{-2}$ white-light illumination, as a function of film thickness for Ti-doped $\alpha-\mathrm{Fe}_{2} \mathrm{O}_{3}$ films on platinized (red stars) or FTO-coated substrates (blue circles). The error bars represent the range of (estimated) systematic errors in the measurements. The red curves show model calculations for films on platinized substrates taking $L=20 \pm 2 \mathrm{~nm}, \vec{P}_{\mathrm{F}} \times \Phi=0.70 \pm 0.05$ and $\overleftarrow{P}_{\mathrm{B}} \times(1-\Phi)=0.25 \pm 0.05$

substrate. This loss highlights the importance of separating the photogenerated holes from the electrons by imposing asymmetric charge transport conditions and blocking the backward injection of holes to the substrate, which is especially critical for ultrathin films wherein a sizeable portion of the photogeneration occurs at the backside of the film ${ }^{28,33}$.

\section{Ultrathin $\alpha-\mathrm{Fe}_{2} \mathrm{O}_{3}$ films on silver-based back reflectors}

To improve the results of our light trapping structures we replaced the lossy platinum reflective coatings by silver-based back reflectors and reduced the backward-injection loss by adding selective electron transport layers between the Ti-doped $\alpha-\mathrm{Fe}_{2} \mathrm{O}_{3}$ films and the substrates. The transition from platinum to silver required developing means to stabilize the back reflectors against tarnishing and thermal etching ${ }^{34}$ during the high-temperature deposition of the $\alpha-\mathrm{Fe}_{2} \mathrm{O}_{3}$ films, as well as against corrosion ${ }^{35}$ during the water splitting tests. The best-performing photoanodes were obtained using $\sim 1.2-\mu \mathrm{m}$-thick layers of silver-gold alloy with 90 at.\% silver and 10 at.\% gold, sandwiched between ultrathin TiN diffusion barrier layers and protected from thermal etching by ultrathin $\mathrm{SnO}_{2}$ over layers (see Supplementary Fig. S6-B). The $\mathrm{SnO}_{2}$ intermediate

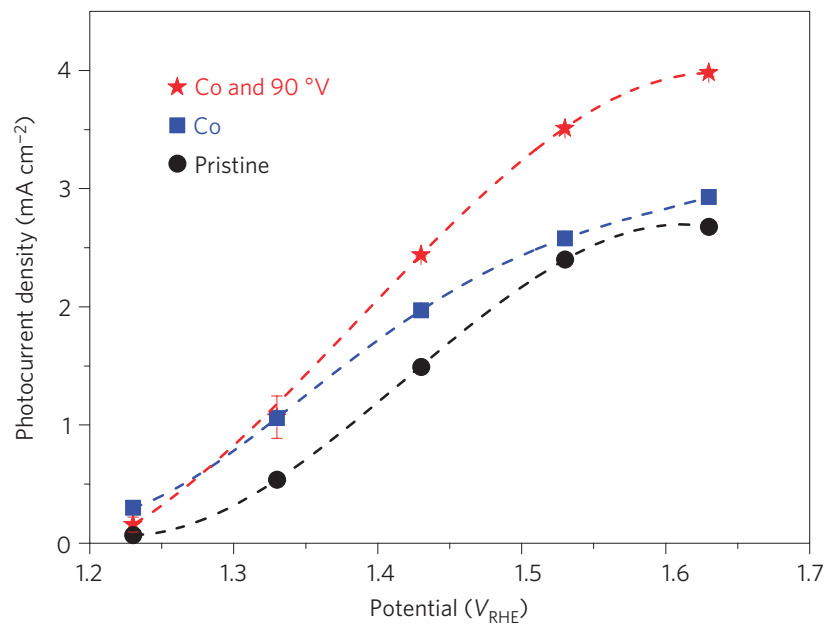

Figure 7 | Water photo-oxidation current densities obtained with ultrathin film $\alpha-\mathrm{Fe}_{2} \mathrm{O}_{3}$ photoanodes on silver-based back reflectors. Steady-state water photo-oxidation current densities obtained using ultrathin $(\sim 26 \mathrm{~nm})$ Ti-doped $\alpha-\mathrm{Fe}_{2} \mathrm{O}_{3}$ films on silver-gold back reflectors, measured in a $1 \mathrm{M}$ $\mathrm{NaOH}$ aqueous solution under exposure to $100 \mathrm{~mW} \mathrm{~cm}^{-2}$ white-light illumination at potentials ranging from 1.23 to $1.63 V_{\text {RHE. }}$ The results for a pristine photoanode with no catalyst are shown as black circles; for a cobalt-treated photoanode as blue squares; and for two cobalt-treated photoanodes placed facing each other in a $90^{\circ} \mathrm{V}$-shaped cell as red stars.

layers also served as selective electron transport layers owing to the large valence-band offset ${ }^{36}$ between $\mathrm{SnO}_{2}$ and $\alpha-\mathrm{Fe}_{2} \mathrm{O}_{3}$ that blocks the injection of holes from the $\alpha-\mathrm{Fe}_{2} \mathrm{O}_{3}$ films to the substrates. These structures were highly reflective (see Supplementary Fig. S3), remaining so through the entire fabrication process (as can be seen by the interference colours in Supplementary Fig. S2-D), and stable against corrosion during the water splitting tests.

With these structures we obtained water photo-oxidation current densities as high as $2.90 \pm 0.01 \mathrm{~mA} \mathrm{~cm}^{-2}$ (at $1.63 V_{\mathrm{RHE}}$, see Supplementary Fig. S22) for a Ti-doped $\alpha-\mathrm{Fe}_{2} \mathrm{O}_{3}$ film deposited using 2,000 laser pulses, yielding a film thickness of $22 \pm 3 \mathrm{~nm}$ (see Supplementary Fig. S6-B). Slightly higher photocurrent densities were obtained using a thicker Ti-doped $\alpha-\mathrm{Fe}_{2} \mathrm{O}_{3}$ film deposited using 2,500 laser pulses (yielding a film thickness of $\sim 26 \mathrm{~nm}$ ) and treated with cobalt catalyst ${ }^{7}$ before the photoelectrochemical measurements. This photoanode reached $3.02 \pm 0.06 \mathrm{~mA} \mathrm{~cm}^{-2}$ at $1.63 V_{\text {RHE }}$ (see Supplementary Fig. S23) and $1.97 \pm 0.01 \mathrm{~mA} \mathrm{~cm}^{-2}$ at $1.43 V_{\text {RHE }}$ (Supplementary Fig. S22). The best results were obtained with two of these photoanodes placed facing each other in a $90^{\circ} \mathrm{V}$-shaped cell ${ }^{30}$ designed to retrap the back-reflected photons (see Supplementary Fig. S21). With this set-up we obtained a photocurrent density of $3.98 \pm 0.03 \mathrm{~mA} \mathrm{~cm}^{-2}$ at $1.63 V_{\mathrm{RHE}}$ (see Supplementary Fig. S24). The results are summarized in Fig. 7.

Resonant light trapping in optical cavities comprising semiconductor ultrathin films on back-reflector substrates enables efficient light absorption in quarter-wave or, in some cases, deeper subwavelength films. This approach overcomes the trade-off between light absorption and collection of photogenerated minority charge carriers that limits the solar energy conversion efficiency of semiconductors with poor transport properties. Taking $\alpha-\mathrm{Fe}_{2} \mathrm{O}_{3}$ photoanodes for water splitting as a case study we predict that as much as $71 \%$ of the solar photons of energy above the bandgap energy of $\alpha-\mathrm{Fe}_{2} \mathrm{O}_{3}$ can be absorbed, at normal incidence, in ultrathin films thinner than $50 \mathrm{~nm}$ wherein most of the photogenerated holes can reach the surface and oxidize water before recombination takes place. The escaped back-reflected photons can be harvested by photon retrapping schemes such as using V-shaped cells, thereby further boosting the light-harvesting 
efficiency. Water photo-oxidation current densities as high as $4 \mathrm{~mA} \mathrm{~cm}^{-2}$ were obtained under exposure to $100 \mathrm{~mW} \mathrm{~cm}^{-2}$ white-light illumination using Ti-doped $\alpha-\mathrm{Fe}_{2} \mathrm{O}_{3}$ ultrathin films on silver-gold-coated back reflectors.

The challenge that our light trapping strategy comes to solve and the underlying physical principles are common to other photoelectrode materials, besides $\alpha-\mathrm{Fe}_{2} \mathrm{O}_{3}$, and other types of solar cell employing semiconductors with poor transport properties. It seems that the enabling key for this strategy to work is strong absorption, as in $\alpha-\mathrm{Fe}_{2} \mathrm{O}_{3}$. Although some semiconductors such as crystalline Si do not meet this prerequisite, many others do. Therefore, the approach can probably be extended to other materials and systems, opening up new opportunities for employing earth-abundant materials that can be produced potentially at a fraction of the cost of the conventional semiconductors employed in photovoltaics technology at present ${ }^{37}$. Furthermore, shrinking the semiconductor films without compromising their light absorption, enabled by our light trapping strategy, holds promising implications for second-generation thin-film solar cells based on CdTe or $\mathrm{CuIn}_{1-x} \mathrm{Ga}_{x} \mathrm{Se}_{2}$ for which the availability of rare-earth elements (Te or In) is a major limitation.

\section{Methods}

See Supplementary Information.

Received 21 September 2011; accepted 4 October 2012; published online 11 November 2012

\section{References}

1. Bard, A. J. \& Fox, M. A. Artificial photosynthesis: Solar splitting of water to hydrogen and oxygen. Acc. Chem. Res. 28, 141-145 (1995).

2. Lewis, N. S. \& Nocera, D. G. Powering the planet: Chemical challenges in solar energy utilization. Proc. Natl Acad. Sci. USA 103, 15729-15735 (2006).

3. Walter, M. G. et al. Solar water splitting cells. Chem. Rev. 110, 6446-6473 (2010)

4. Fujishima, A. \& Honda, K. Electrochemical photolysis of water at a semiconductor electrode. Nature 238, 37-38 (1972).

5. Wrighton, M. S. Photoelectrochemical conversion of optical energy to electricity and fuels. Acc. Chem. Res. 12, 303-310 (1979).

6. Rajeshwar, K. Hydrogen generation at irradiated oxide semiconductor-solution interfaces. J. Appl. Electrochem. 37, 765-787 (2007).

7. Kay, A., Cesar, I. \& Grätzel, M. New benchmark for water photooxidation by nanostructured $\alpha-\mathrm{Fe}_{2} \mathrm{O}_{3}$ Films. J. Am. Chem. Soc. 128, 15714-15721 (2006).

8. Van de Krol, R., Liang, Y. \& Schoonman, J. Solar hydrogen production with nanostructured metal oxides. J. Mater. Chem. 18, 2311-2320 (2008).

9. Sivula, K., Le Formal, F. \& Grätzel, M. Solar water splitting: Progress using hematite $\left(\alpha-\mathrm{Fe}_{2} \mathrm{O}_{3}\right)$ photoelectrodes. ChemSusChem 4, 432-449 (2011).

10. Chen, Z. et al. Accelerating materials development for photoelectrochemical hydrogen production: Standards for methods, definitions, and reporting protocols. J. Mater. Res. 25, 3-16 (2010).

11. Brillet, J. et al. Examining architectures of photoanode-photovoltaic tandem cells for solar water splitting. J. Mater. Res. 25, 17-24 (2010).

12. Tilley, S. D., Cornuz, M. \& Sivula, K. Light-induced water splitting with hematite: Improved nanostructure and iridium oxide catalysis. Angew. Chem. Int. Ed. 49, 6405-6408 (2010).

13. Dare-Edwards, M. P., Goodenough, J. B., Hamnett, A. \& Trevellick, P. R. Electrochemistry and photoelectrochemistry of iron(III) oxide. J. Chem. Soc. Faraday Trans. 79, 2027-2041 (1983).

14. Kennedy, J. H. \& Frese, K. W. Jr Photooxidation of water at $\alpha-\mathrm{Fe}_{2} \mathrm{O}_{3}$ electrodes. J. Electrochem. Soc. 125, 709-714 (1978).

15. Wheeler, D. A., Wang, G., Ling, Y., Li, Y. \& Zhang, J. Z. Nanostructured hematite: Synthesis, characterization, charge carrier dynamics, and photoelectrochemical properties. Energy Environ. Sci. 5, 6682-6702 (2012).

16. Gärtner, W. W. Depletion layer photoeffects in semiconductors. Phys. Rev. 116, 84-87 (1959).

17. Dotan, H., Sivula, K., Grätzel, M., Rothschild, A. \& Warren, S. C. Probing the photoelectrochemical properties of hematite $\left(\alpha-\mathrm{Fe}_{2} \mathrm{O}_{3}\right)$ electrodes using hydrogen peroxide as a hole scavenger. Energy Environ. Sci. 4, 958-964 (2011).

18. Upul Wijayantha, K. G., Saremi-Yarahmadia, S. \& Peter, L. M. Kinetics of oxygen evolution at $\alpha-\mathrm{Fe}_{2} \mathrm{O}_{3}$ photoanodes: A study by photoelectrochemical impedance spectroscopy. Phys. Chem. Chem. Phys. 13, 5264-5270 (2011).
19. Pendlebury, S. R. et al. Correlating long-lived photogenerated hole populations with photocurrent densities in hematite water oxidation photoanodes. Energy Environ. Sci. 5, 6304-6312 (2012).

20. Klahr, B. M. \& Hamann, T. W. Current and voltage limiting processes in thin film hematite electrodes. J. Phys. Chem. C 115, 8393-8399 (2011).

21. Kumar, A., Santangelo, P. G. \& Lewis, N. S. Electrolysis of water at $\mathrm{SrTiO}_{3}$ photoelectrodes: Distinguishing between the statistical and stochastic formalisms for electron-transfer processes in fuel-forming photoelectrochemical systems. J. Phys. Chem. 96, 834-842 (1992).

22. Klahr, B. M., Martinson, A. B. F. \& Hamann, T. W. Photoelectrochemical investigation of ultrathin film iron oxide solar cells prepared by atomic layer deposition. Langmuir 27, 461-468 (2011).

23. Yablonovitch, E. \& Cody, G. D. Intensity enhancement in textured optical sheets for solar cells. IEEE Trans. Electron Dev. 29, 300-305 (1982).

24. Yablonovitch, E. Statistical ray optics. J. Opt. Soc. Am. 72, 899-907 (1982).

25. Bockris, J. O’M. \& Uosaki, K. The theory of the light-induced evolution of hydrogen at semiconductor electrodes. J. Electrochem. Soc. 125, 223-227 (1978)

26. Pettersson, L. A. A., Roman, L. S. \& Inganäs, O. Modeling photocurrent action spectra of photovoltaic devices based on organic thin films. J. Appl. Phys. 86, 487-496 (1999)

27. Born, M. \& Wolf, E. Principles of Optics 7th edn (Cambridge Univ. Press, 2010).

28. Formal, F. L., Grätzel, M. \& Sivula, K. Controlling photoactivity in ultrathin hematite films for solar water-splitting. Adv. Funct. Mater. 20, 1099-1107 (2010).

29. Murphya, A. B. et al. Efficiency of solar water splitting using semiconductor electrodes. Int. J. Hydrogen Energ. 31, 1999-2017 (2006).

30. Rim, S. B., Zhao, S., Scully, S. R., McGehee, M. D. \& Peumans, P. An effective light trapping configuration for thin-film solar cells. Appl. Phys. Lett. 91, 243501 (2007).

31. Würfel, P. Physics of Solar Cells 2nd edn (Wiley, 2009).

32. Klahr, B., Gimenez, S., Fabregat-Santiago, F., Bisquert, J. \& Hamann, T. W. Electrochemical and photoelectrochemical investigation of water oxidation with hematite electrodes. Energy Environ. Sci. 5, 7626-7636 (2012).

33. Hisatomi, T. et al. Enhancement in the performance of ultrathin hematite photoanode for water splitting by an oxide underlayer. Adv. Mater. 24, 2699-2702 (2012).

34. Chalmers, B., King, R. \& Shuttleworth, R. The thermal etching of silver. Proc. R. Soc. Lond. Ser. A Math. Phys. Sci. 193, 465-483 (1948).

35. Pourbaix, M. Atlas of Electrochemical Equilibria in Aqueous Solutions 2nd edn (NACE, 1974).

36. Grätzel, M. Photoelectrochemical cells. Nature 414, 338-344 (2001).

37. Wadia, C., Alivisatos, A. P. \& Kammen, D. M. Materials availability expands the opportunity for large-scale photovoltaics deployment. Environ. Sci. Technol. 43, 2072-2077 (2009).

\section{Acknowledgements}

This work was supported by the European Commission Seventh Framework Programme (NanoPEC, project 227179), by the I-CORE Program of the Planning and Budgeting Committee and The Israel Science Foundation (grant No. 152/11) and by the KAMIN project from the Office of the Chief Scientist (OCS) in the Ministry of Industry, Trade and Labor (MOITAL). The authors thank S. C. Warren, G. Bartal and $\mathrm{N}$. Tessler for reading the manuscript and providing useful comments and suggestions for improving it.

\section{Author contributions}

H.D. and O.K. developed the optical simulation model, and H.D. and A.R. added to it the charge transport model. H.D. designed and fabricated the photoelectrodes and carried out most of the optical and photoelectrochemical measurements. E.S. and O.B. developed the stable silver-gold alloy back reflectors. H.D. and M.G. developed the selective electron transport hole blocking under layers. I.D. carried out the cross-section transmission electron microscope measurements. G.A. carried out the spectroscopic ellipsometry measurements and analysis. A.R. supervised the project and wrote the manuscript. All authors discussed the manuscript and agreed on its final content.

\section{Additional information}

Supplementary information is available in the online version of the paper. Reprints and permissions information is available online at www.nature.com/reprints. Correspondence and requests for materials should be addressed to A.R.

\section{Competing financial interests}

The authors declare no competing financial interests. 